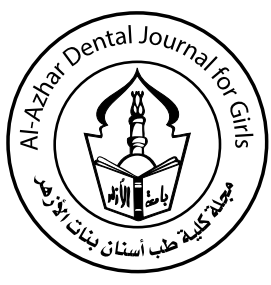

\title{
Evaluation of the Treatment Outcomes for Class II Malocclusion by Using PowerScope Appliance
}

\author{
MennatAllah M. Shendy ${ }^{(1)}$, Samir A. Ibrahim ${ }^{(2)}$ and Ahmed E. Salama ${ }^{(3)}$
}

Codex : 47/1710

dentaljournal.forgirls@yahoo.com

\section{KEYWORDS}

Class II malocclusion, non-extraction, fixed functional appliance, PowerScope appliance.

\begin{abstract}
Objective: This study was designed to evaluate the treatment outcomes for Class II malocclusion by using PowerScope Appliance. Patients, materials and methods: A sample of 10 patients with an age range from 14 to 18 years, suffering from skeletal Class 2 and Angle Class II division 1 malocclusion. All patients didn't undergo any orthodontic treatment previously and were free of systemic diseases that affect craniofacial growth. Pre and post-treatment lateral cephalograms were obtained, cephalometric analysis was performed. Class I relation were obtained in with a substantial improvement in facial profile, skeletal jaw relationship, and overall esthetic appearance of the patient. A significant forward displacement of the mandible was the principal element for successful correction of Class II malocclusion. Results: Restriction of maxillary growth with significant mandibular growth were observed with PowerScope appliance. The Class II correction was obtained mainly by slight maxillary molar distalization and intrusion, in addition to mesial migration of the mandibular molars and flaring of the lower incisors. Soft tissue profile improvements was observed with this appliance. Conclusions: PowerScope appliance provides an effective correction of Class II division 1 malocclusion in adolescent patients as it promotes restriction of anterior maxillary displacement with significant forward mandibular repositioning which reduces both skeletal and soft tissue profile convexities. PowerScope appliance caused dentoalveolar changes including slight maxillary molar distalization, in addition to mesialization of the lower molars and proclination of the lower incisors which results in correction of Class II malocclusion.
\end{abstract}

\section{INTRODUCTION}

One of the most common orthodontic problems encountered in the orthodontic practice is Class 2 malocclusion. This type of discrepancy

- Paper extracted from Master thesis entitled "Evaluation of the Treatment Outcomes for Class II Malocclusion by Using PowerScope Appliance".

1. Dentist at Ministry of health.

2. Professor and Head of Orthodontic Department, Faculty of Dental Medicine for Girls, Al-Azhar University.

3. Professor of Orthodontics, Orthodontic Department, Faculty of Dental Medicine for Girls, Al-Azhar University. 
causes change in skeletal profile such as mandibular retrusion, maxillary protrusion or a combination of both. ${ }^{(1)}$ Many treatment modalities were proposed for such cases like functional appliances which may be removable such as Harvold activator, bionator, Twin block or fixed such as Herbst, Jasper Jumper, Advansync and PowerScope. ${ }^{(2-4)}$ Emil Herbst presented the first fixed bite jumping device for Class II treatment In 1909, it is still popular but has the disadvantage of being rigid so it limits lateral mandibular movements and oral hygiene maintenance ${ }^{(5)}$. Evolution of fixed functional appliances over years led to introduction of newer appliances with better results in noncompliant patients. One of these innovations is PowerScope appliance which is a hybrid appliance for the correction of mild skeletal class II malocclusion in noncompliant patients ${ }^{(6) .}$

\section{PATIENTS, MATERIALS AND METHODS}

This study was performed on 10 patients, 4 males and 6 females with Class II malocclusion of age range from 14 to 18 years. All participants underwent bonding of fixed orthodontic appliance (Unitek $^{\mathrm{TM}}$ Metal bands, 3M Unitek USA, Unitek ${ }^{\mathrm{TM}}$ Miniature Twin Metal Brackets, 3M, Unitek USA, Unitek $^{\mathrm{TM}}$ Orthodontic Composite, 3M, Unitek USA).Sequential orthodontic arch wires were used starting from 0.012 " $\mathrm{Ni}$-Ti up to $0.019 \times 0.025$ " St.St. arch wire (G \& H wire company, USA). The PowerScope appliance was used until the patient's mandible could not be manipulated more posterior than one $\mathrm{mm}$ overjet inter-dental relationship and molar relation and canine relation are corrected to Class I, Then appliance was removed and a new lighter stainless steel arch wire placed, the patient was instructed to use 3/16" heavy inter-maxillary elastics for three months from the maxillary canines and first premolars to the mandibular first and second premolars for posterior occlusion settling.

The patients and/or guardians were fully informed about the procedures, and informed written consents were obtained before commencing the study and approved by the Ethical Committee of Faculty of Dental Medicine for Girls, Al-Azhar University.

The following routine orthodontic records were obtained for each patient before treatment:

1. Extra-oral photographs (frontal at rest, frontal during smile, right and left profile views).

2. Intra-oral photographs (frontal, right and left side views, upper and lower occlusal views).

3. Orthodontic study models.

4. Standardized lateral cephalometric radiographs.

5. Panoramic radiograph.

In addition, post-PowerScope extra oral photographs, intra oral photographs and lateral cephalograms were obtained after an average observation period of 6 to 8 months then skeletal, dental and soft tissue measurements were evaluated after cephalometric analysis.

The collected numerical data were explored for normality by checking the data distribution, using Kolmogorov-Smirnov and Shapiro-Wilk tests. All data showed parametric (normal) distribution except for 1U-NA, 1U-NA (mm), E-line to U lip, E-line to L lip, S-line to U lip and S-line to L lip. Data were represented as mean, standard deviation (SD), median, range and $95 \%$ Confidence interval (95\% CI) values. For parametric data; Paired t-test was used to study the changes after treatment. For non-parametric data; Wilcoxon signed-rank test was used to study the changes after treatment. Qualitative data were presented as frequencies and percentages.

The significance level was set at $\mathrm{P} \leq 0.05$. Statistical analysis was performed with IBM® SPSS ${ }^{\circledR}$ Statistics Version 20 for Windows (IBM Corporation, NY, USA and SPSS, Inc., an IBM Company).

\section{RESULTS}

The results of the study showed that there was a statistically significant decrease in mean $\mathrm{SNA}^{\circ}$, 
$\mathrm{ANB}^{\circ}$ and $\mathrm{N}-\mathrm{A}-\mathrm{Pg}^{\mathrm{o}}$ post-treatment and statistically significant increase in mean $\mathrm{SNB}^{\circ}, \mathrm{S}-\mathrm{Ar}^{-\mathrm{Go}^{\circ}}$ and Facial angle post-treatment. Regarding the linear skeletal measurements there was statistically significant increase in mean ramus height, $\mathrm{PFH}(\mathrm{mm})$ and total mandibular length post-treatment. But there was no statistically significant change in mean LAFH (mm), LAFH/TAFH. (Tables 1\&2).

Concerning the dental angular and linear measurements. There was a statistically significant decrease in mean $1 \mathrm{U}_{-\mathrm{SN}}{ }^{\circ}, 1 \mathrm{U}_{-\mathrm{NA}}{ }^{\circ}$ and $\mathrm{IL}_{-\mathrm{SN}}{ }^{\circ}$ post-treatment and statistically significant increase in mean $1 \mathrm{~L}-\mathrm{NB}^{\circ}$ and $1 \mathrm{U}-1 \mathrm{~L}^{\circ}$ post-treatment while there was a a statistically significant decrease in mean 1U-NA (mm), IL-SN, 6L-NB (mm), overjet and overbite post-treatment. There was a statistically significant increase in mean 6U-NA $(\mathrm{mm})$, 1L-NB (mm) post-treatment. (Tables3\&4)

The results of angular soft tissue measurement showed that there was a statistically significant increase in mean $\mathrm{H}$ angle post-treatment. (Table 5)

Table (1) Mean, standard deviation (SD) values and results of paired t-test for the changes in skeletal angular measurements after treatment

\begin{tabular}{|c|c|c|c|c|c|c|c|}
\hline \multirow{2}{*}{$\begin{array}{c}\text { Skeletal angular } \\
\text { measurements }\end{array}$} & \multicolumn{2}{|c|}{ Pre-treatment } & \multicolumn{2}{c|}{ Post-treatment } & \multicolumn{2}{c|}{ Change } & \multirow{2}{*}{$P$-value } \\
\cline { 2 - 7 } & Mean & SD & Mean & SD & Mean & SD & \\
\hline SNA $^{\circ}$ & 82.10 & 4.20 & 81.40 & 3.81 & -0.70 & 0.67 & $0.010^{*}$ \\
\hline SNB $^{\circ}$ & 74.40 & 3.53 & 77.50 & 3.34 & 3.10 & 0.46 & $<0.001^{*}$ \\
\hline ANB $^{\circ}$ & 7.70 & 1.25 & 3.90 & 0.81 & -3.80 & 0.71 & $<0.001^{*}$ \\
\hline $\mathrm{N}-A-P g^{\circ}$ & 13.10 & 4.81 & 8.05 & 2.95 & -5.05 & 2.30 & $<0.001^{*}$ \\
\hline $\mathrm{S}^{\circ}$ Ar-Go & 147.85 & 10.53 & 150.65 & 10.42 & 2.80 & 1.03 & $<0.001^{\circ}$ \\
\hline Facial angle & 85.70 & 3.68 & 90.75 & 2.84 & 5.05 & 2.01 & $<0.001^{*}$ \\
\hline
\end{tabular}

*: Significant at $P \leq 0.05 \quad$ Sample size $=10$ patients

Table (2) Mean, standard deviation (SD) values and results of paired t-test for the changes in skeletal linear measurements after treatment

\begin{tabular}{|c|c|c|c|c|c|c|c|}
\hline \multirow{2}{*}{ Skeletal linear measurements } & \multicolumn{2}{|c|}{ Pre-treatment } & \multicolumn{2}{|c|}{ Post-treatment } & \multicolumn{2}{|c|}{ Change } & \multirow{2}{*}{$P$-value } \\
\hline & Mean & SD & Mean & $\mathrm{SD}$ & Mean & SD & \\
\hline UAFH (mm) & 50.90 & 3.07 & 49.45 & 2.83 & -1.45 & 1.21 & $0.004 *$ \\
\hline LAFH (mm) & 66.85 & 8.24 & 66.05 & 8.82 & -0.80 & 1.90 & 0.216 \\
\hline TAFH (mm) & 114.50 & 8.13 & 111.80 & 7.91 & -2.70 & 2.11 & $0.003 *$ \\
\hline PFH (mm) & 70.10 & 7.89 & 72.55 & 6.52 & 2.45 & 2.24 & $0.007 *$ \\
\hline LAFH/TAFH (mm) & 58.50 & 4.30 & 59.53 & 5.18 & 1.03 & 1.55 & 0.065 \\
\hline Ramus height (mm) & 58.30 & 8.72 & 59.40 & 8.85 & 1.10 & 0.99 & 0.007 * \\
\hline Corpus length (mm) & 65.30 & 4.27 & 64.40 & 5.11 & -0.90 & 2.22 & 0.232 \\
\hline Total mandibular length (mm) & 103.20 & 8.05 & 106.20 & 8.16 & 3.00 & 2.00 & $0.001 *$ \\
\hline
\end{tabular}

*: Significant at $P \leq 0.05$

Sample size $=10$ patients 
Table (3): Mean, standard deviation (SD) values and results of paired t-test and Wilcoxon signed-rank test for the changes in dental angular measurements after treatment

\begin{tabular}{|l|c|c|c|c|c|c|c|}
\hline \multirow{2}{*}{ Dental angular measurements } & \multicolumn{2}{|c|}{ Pre-treatment } & \multicolumn{2}{|c|}{ Post-treatment } & \multicolumn{2}{c|}{ Change } & \multirow{2}{*}{$P$-value } \\
\cline { 2 - 8 } & Mean & SD & Mean & SD & Mean & SD & \\
\hline $1 \mathrm{U}-S N^{\circ}$ & 109.90 & 5.95 & 94.30 & 5.85 & -15.60 & 4.33 & $<0.001^{*}$ \\
\hline $1 \mathrm{U}-N A^{\circ}$ & 28.30 & 6.78 & 12.25 & 6.12 & -16.05 & 4.21 & $0.005^{*}$ \\
\hline $1 \mathrm{~L}-S N^{\circ}$ & 41.90 & 5.11 & 38.70 & 6.27 & -3.20 & 4.29 & $0.043^{*}$ \\
\hline $1 \mathrm{~L}-N B^{\circ}$ & 32.85 & 6.23 & 36.70 & 5.54 & 3.85 & 3.74 & $0.010^{*}$ \\
\hline $1 \mathrm{U}^{\circ} 1 \mathrm{~L}^{\circ}$ & 113.70 & 5.93 & 123.70 & 3.43 & 10.00 & 7.35 & $<0.001^{*}$ \\
\hline
\end{tabular}

*: Significant at $P \leq 0.05$

Sample size $=10$ patients

Table (4): Mean, standard deviation (SD) values and results of paired t-test and Wilcoxon signed-rank test for the changes in dental linear measurements after treatment

\begin{tabular}{|l|c|c|c|c|c|c|c|}
\hline \multirow{2}{*}{ Dental linear measurements } & \multicolumn{2}{|c|}{ Pre-treatment } & \multicolumn{2}{c|}{ Post-treatment } & \multicolumn{2}{c|}{ Change } & \multirow{2}{*}{$P$-value } \\
\cline { 2 - 8 } & Mean & SD & Mean & SD & Mean & SD & \\
\hline 1U-NA $(\mathrm{mm})$ & 7.04 & 2.67 & 4.35 & 2.65 & -2.69 & 0.75 & $0.005^{*}$ \\
\hline 6U-NA $(\mathrm{mm})$ & 21.95 & 1.79 & 23.90 & 2.33 & 1.95 & 1.32 & $0.001^{*}$ \\
\hline 1L-NB $(\mathrm{mm})$ & 7.32 & 2.11 & 8.69 & 1.89 & 1.37 & 0.38 & $<0.001^{*}$ \\
\hline 6L-NB $(\mathrm{mm})$ & 17.60 & 2.50 & 13.90 & 2.32 & -3.70 & 1.84 & $0.002^{*}$ \\
\hline Overjet $(\mathrm{mm})$ & 9.50 & 2.00 & 1.25 & 0.37 & -8.25 & 1.77 & $<0.001^{*}$ \\
\hline Overbite $(\mathrm{mm})$ & 4.50 & 0.85 & 1.12 & 0.32 & -3.38 & 0.84 & $<0.001^{*}$ \\
\hline
\end{tabular}

*: Significant at $P \leq 0.05$

Sample size $=10$ patients

Table (5): Mean, standard deviation (SD) values and results of paired t-test for the changes in soft tissue angular measurements after treatment

\begin{tabular}{|c|c|c|c|c|c|c|c|}
\hline \multirow{2}{*}{ Soft tissue angular measurements } & \multicolumn{2}{|c|}{ Pre-treatment } & \multicolumn{2}{|c|}{ Post-treatment } & \multicolumn{2}{|c|}{ Change } & \multirow{2}{*}{$P$-value } \\
\cline { 2 - 7 } & Mean & SD & Mean & SD & Mean & SD & \\
\hline H angle & 21.40 & 2.07 & 24.10 & 1.31 & 2.70 & 0.86 & $<0.001^{*}$ \\
\hline
\end{tabular}

*: Significant at $P \leq 0.05$

Sample size $=10$ patients

\section{DISCUSSION}

Angle Class II division 1 malocclusion has been considered one of the most encountered problems in the orthodontic practice. Class II division 1 malocclusion affects about $16.2 \%$ of all Egyptian subjects seeking orthodontic treatment with variable combinations of dental and skeletal factors contributing to the disharmony. For single phase treatment of the malocclusion in the adolescent period, various treatment strategies exist including the use of headgear associated with a fixed appliance with the adjunct of Class II elastics. An alternative approach is functional jaw orthopedics immediately followed by fixed appliances to refine the occlusion. 
There is an increasing interest in noncompliance appliances that allow treatment to be done without depending on co-operation from patient or parents. This definition of noncompliance appliances excludes removable devices of any sort.

The re-introduction of Herbst appliance by Pancherz in the late $70 \mathrm{~s}{ }^{(4)}$ led to the introduction and invention of many different types of fixed functional appliances, some of which are rigid as the MARA and AdvanSync appliances, others are flexible as Jasper Jumper while others are hybrid with some features of the rigid appliances and other features of the flexible ones. PowerScope is a fixed push type appliance clamped bilaterally to the upper and lower archwires. Each unit is made of cylinder housing nickel titanium coil springs. Two Hex nuts are present, one attaching the appliance to the upper archwire mesial to the maxillary permanent first molar and the other one distal to the mandibular canine. A constant force of approximately $260 \mathrm{gm}$ is delivered on each side at full compression ${ }^{(7-8)}$.The purpose of that study was to evaluate the skeletal, dental and soft tissue effects produced by PowerScope Class II corrector in treatment of Class II division 1 subjects. The sample of the study consisted of 10 subjects with age range from 14 to 18 years. All patients were treated from six to eight months as recommended by other authors investigating PowerScope appliance ${ }^{(8,9)}$.

\section{Results' interpretation:}

\section{Regarding the skeletal changes}

In the current study; PowerScope Class II Corrector resulted in significant effect on maxilla as indicated by $(P \leq 0.05)$ decrease in the SellaNasion-A point angle. This effect is often observed with the use of fixed functional appliances, similar finding in other studies was reported after using PowerScope in treatment of Class II malocclusion ${ }^{(6,7)}$ significant reduction in SNA was also reported with the use of Herbst appliance ${ }^{(10)}$ as well as with Jasper Jumper ${ }^{(11)}$ and after treatment with AdvanSync ap- pliance $^{(12,13)}$ Regarding the mandible there was significant anterior displacement demonstrated by statistically significant increase in SNB $(P<0.001)$, Similar finding was reported in other studies using PowerScope Class II corrector ${ }^{(6-9)}$ same results were demonstrated with TFBC $^{(14-16)}$ and also with Herbst appliance $^{(5,10,22)}$ and with mandibular protraction appliance $^{(17)}$. However other studies demonstrated different results as with MARA ${ }^{(18)}$. Regarding the sagittal intermaxillary jaw relationship, it was improved as evidenced by a highly significant reduction value for the ANB with $(P<0.001)$ between pre and post treatment results. Same result was found by other authors investigating PowerScope appliance (7-9) Regarding the facial convexity (N-A-Pg) there was a significant reduction by a $(P<0.001)$, the improvement of the facial convexity is a result of backward movement of the A point and forward movement of the pogonion. Similar results were reported in other studies dealing with Herbst appliance ${ }^{(19)}$

\section{Regarding the dento-alveolar changes:}

The result of the current study showed significant retroclination of the upper incisor as upper incisor angulation to SN plane significantly decrease. Also upper incisor inclination to NA and upper incisor position relative to NA were significantly decreased. These results agreed with other studies on PowerScope ${ }^{(6,8)}$.Similar results were reported with TFBC $^{(14-16)}$ with AdvanSync ${ }^{(12,13)}$ and with Eureka Spring ${ }^{(20)}$.

Upper molar showed significant distalization which appeared in significant increase in distance between mesial cusp of U6 to NA. Similar results were obtained with $\mathrm{TFBC}^{(16)}$.On the other hand Chhibber et al reported that upper molars underwent mesial movement with TFBC treatment and explained that such finding might be attributed to the mechanics involved during finishing of treatment after removal of FFA or natural tendency of teeth to drift mesially ${ }^{(14)}$. 
Regarding the lower dentition, lower incisors showed significant proclination presented by significant increase in lower incisor inclination relative to NB plane and significant increase in lower incisor position relative to NB plane with $P$ values $(0.010 \&$ $<0.001)$ respectively. Those findings were in agreement with the results reported by Khumanthem et $\mathrm{al}^{(6)}$ and Paulose et $\mathrm{al}^{(7,9)}$ with the use of PowerScope appliance also with the use of $\mathrm{TFBC}^{(16)}$ as well as in results obtained with Herbst appliance ${ }^{(5)}$ and with Eureka Spring ${ }^{(20)}$.

Lower molars showed significant mesialization presented by significant reduction in the distance between mesial surface of L6 and NB plane with $P=0.002$, Similar results were obtained in a study on PowerScope ${ }^{(7,9)}$ and also by other studies using Jasper Jumper appliance ${ }^{(23,24)}$.

Regarding the Overjet, PowerScope Class II corrector resulted in significant decrease in the overjet with $(P<0.001)$, the reduction in the overjet was achieved by both skeletal and dental changes in the sagittal direction in which there was restriction in forward movement of the A-point with retroclination of the upper incisors and forward movement of B-point with mandibular incisors proclination. Similar results were found in previous studies on PowerScope ${ }^{(6,8,9)}$, Almost all FFAs showed significant decrease in the overjet.

Significant decrease of mean $-5 \mathrm{~mm}$ in the overjet was reported with Herbst appliance ${ }^{(21)}$ and MPA-IV showed marked reduction in overjet ${ }^{(25)}$, also studies on AdvanSync appliance reported significant overjet reduction ${ }^{(12,13)}$. Jasper Jumper appliance resulted in significant ovejet reduction ${ }^{(23,24)}$.

Regarding the overbite, PowerScope Class II Corrector resulted in significant reduction in the overbite from about $65 \%$ to $30 \%$ with a $P$-value $<0.001$. This reduction in overbite might be attributed to proclination of the lower incisors in combination with the anterior edge to edge relationship which left the buccal segment out of occlusion and gave the chance for normal buccal segment vertical growth. Studies on TFBC reported reduction in the overbite with a mean value of $-3.2 \mathrm{~mm}{ }^{(15,16)}$ which is very close to the results of this study. Significant decrease for the overbite with a mean value $-2.4 \mathrm{~mm}$ was reported from a study on Herbst appliance ${ }^{(21,22)}$ and another study on Jasper Jumper appliance (24) Regarding the inter-incisal angle, this study showed significant increase with a mean of $10^{\circ}$. This could be due to decrease in the maxillary incisors inclination more than mandibular incisors proclination. Same results were obtained in studies investigating the effect of PowerScope ${ }^{(6,8,9)}$.Similar increase in the interincisal angle was reported with $\mathrm{TFBC}^{(16)}$ and MARA appliance ${ }^{(18)}$.

\section{Regarding the dento-alveolar changes:}

In the contemporary study at the end of treatment with PowerScope appliance, it was improved as the results showed a high statistically significant reduction, evident by the significant increase at the $\mathrm{H}$-angle which might be attributed to the forward movement of the chin that is reflected due to mandibular advancement. Similar results were reported with PowerScope in other studies ${ }^{(6,8,9)}$ as well as with TFBC $^{(14,16)}$. Nearly all studies on FFAs that reported its effect on soft tissues revealed the same ${ }^{(10,22)}$

The upper lip showed significant retrusion with PowerScope appliance in this study as showed by significant decrease in measurement of the horizontal distance between upper lip and E-line. Similar significant retrusion of upper lip was reported in a case study on PowerScope, also similar significant retrusion of upper lip was reported by Pancherz ${ }^{(22)}$ in the study that used Herbst appliance and with Jasper Jumper appliance ${ }^{(11)}$.

The lower lip presented significant protrusion with PowerScope appliance as showed by the significant increase in measurement of the horizontal distance between lower lip and S-line as well as E-line, while statistical values were insignificant. Similar protrusion of lower lip was evident with the use of Herbst appliance ${ }^{(22)}$ and with mandibular protrac- 
tion appliance ${ }^{(17)}$ as well as in a comparative study between AdvanSync appliance and intermaxillary elastics ${ }^{(13)}$, TFBC appliance ${ }^{(14)}$ revealed same result as for distance between lower lip and S-line and significant anterior movement of lower lip towards the E-line was also reported in a study on TFBC ${ }^{(16)}$.

\section{CONCLUSIONS}

On the basis of the current results of the present study, the following conclusions could be drawn:

1. PowerScope appliance provides an effective correction of Class II division 1 malocclusion in adolescent patients.

2. The PowerScope appliance promotes restriction of anterior maxillary displacement with significant forward mandibular repositioning which reduces both skeletal and soft tissue profile convexities.

3. PowerScope appliance caused dentoalveolar changes including slight maxillary molar distalization, in addition to mesialization of the lower molars and proclination of the lower incisors which results in correction of Class II malocclusion.

\section{REFERENCES}

1. Graber TM, Vanarsdall RL and Vig KW. Orthodontics Current Principles and Techniques. Mosby. 2005; 2:493-6.

2. Wahl N. Orthodontics in 3 millennia. Chapter 9: Functional appliances to midcentury. Am J Orthod Dentofac Orthop. 2006; 129:829-33.

3. McNamara J, Peterson J, Alexander R. Three-Dimensional Diagnosis and Management of Class II Malocclusion in the Mixed Dentition. Semin Orthod. 1996; 2:114-37.

4. Ritto AK. Fixed Functional Appliances - A Classification. Orthod CYBER J. 2001.

5. Pancherz H. History, Background and development of the Herbst Appliance. Semin Orthod. 2003; 9:3-11.

6. Khumanthem S, Kumar M, Ansari A, Jain A. Correction of Class II using PowerScope Appliance - A Case Report. Arch of Dent and Med Res 2016; 2:120-5.
7. Moro A. Simplified Correction of Class II Using PowerScope -A case report. Clinical orthodontics 2016; 2:1-27.

8. Paulose J, Antony PJ, Sureshkumar B, George SM, Mathew MM, Sebastian J. PowerScope a Class II corrector-A case report. Contemp Clin Dent 2016; 7:2215.

9. Nishanth B, Gopinath A, Ahmed S, Patil N, Srinivas K, Chaitanya A. Cephalometric and computed tomography evaluation of dentoalveolar/soft-tissue change and alteration in condyle-glenoid fossa relationship using the PowerScope: A new fixed functional appliance for Class II correction -A clinical study. Int J Orthod Rehabil 2017;8:41-50.

10. Booij JW, Goeke J, Bronkhorst EM. Class II treatment by extraction of maxillary first molars or Herbst appliance: dentoskeletal and soft tissue effects in comparison. J Orofac Orthop 2013; 74:52-63.

11. Küçükkeleş N, İlhan Iş, Orgun İ A .Treatment Efficiency in Skeletal Class II Patients Treated with the Jasper Jumper. Angle Orthod. 2007; 77:3,449-56.

12. Al-Jewair TS, Preston CB, Moll EM, Dischinger T. A comparison of the MARA and the AdvanSync functional appliances in the treatment of Class II malocclusion. Angle Orthod. 2012; 82:907-14.

13. Jayachandran S, Wiltshire WA, Hyasaki SM. Comparison of AdvanSync and intermaxillary elastics in the correction of Class II malocclusions: A retrospective clinical study. Am J Orthod Dentofac Orthop. 2016;150:979-988.

14. Chhibber A, Upadhyay M,Uribe F, Nanda R. Mechanism of Class II correction in prepubertal and postpubertal patients with Twin Force Bite Corrector. Angle Orthod.2013; 83:718-27.

15. GuimarãesJr CH, Henriques JFC, Janson G, Rodrigues de Almeida M, Araki J, Cançado RH, Castro R, and Nanda R. Prospective study of dentoskeletal changes in Class II division malocclusion treatment with twin force bite corrector. Angle Orthod. 2013; 83:319-26.

16. Abdelwahed AM. Evaluation of Soft and Hard Tissue Changes Concomitant to Twin Force Bite Corrector Therapy in Class II Malocclusion. Master thesis, Al-Azhar University Girls’ Branch, 2014. 
17. Siqueira DF, Almeira RR, Janson G, et al. Dentoskeletal and soft tissue changes with cervical headgear and mandibular protraction appliance therapy in the treatment of class II malocclusion. Am Orthod Dentofac Orthop. 2007; 131:447.

18. Marcelo N, Pangrazio K, Pangrazio-Kulbersh V, Berger JL, Bayirli B. Treatment effects of the mandibular anterior repositioning appliance in patients with Class II skeletal malocclusions. Angle Orthod. 2012; 82:971-77.

19. Pancherz H. Tretament of class II malocclusion by jumping the bite with the Herbst appliance. Am J Orthod. $1979 ; 76: 423-42$.

20. Stromeyer EL, Caruso JM, and DeVincenzo JP. A Cephalometric Study of the Class II Correction Effects of the Eureka Spring. Angle Orthod. 2002; 72:203- 10.

21. Omblus J, Malmgren O, Pancherz H, Hägg U, Hansen K. Long-term effects of Class II correction in Herbst and Bass therapy. Eur J Orthod. 1997; 19: 185-93.

22. Ruf $S$ and Pancherz H. Herbst/multibracket appliance treatment of class II division 1 malocclusion in early and late adulthood. Eur J Orthod. 2006; 28:352-60.

23. Santiago de Lima KJR, Henriques JFC, Janson G, Pereira SCC, Neves LS, Cançado RH. Dentoskeletal changes induced by the Jasper jumper and the activator-headgear combination appliances followed by fixed orthodontic treatment. Am J Orthod and Dentofac Orthop. 2013; 143:684-94.

24. Bassarelli T, Franchi L, Defraia E, Melsen B. Dentoskeletal effects produced by a Jasper Jumper with an anterior bite plane. Angle Orthod. In-Press, 2016.

25. Jena AK and Duggal R. Treatment Effects of Twin-Block and Mandibular Protraction Appliance IV in the Correction of Class II Malocclusion. Angle Orthod. 2010; 80:485-91. 